\title{
Mechanical fibrinogen-depletion supports heparin-free mesenchymal stem cell propagation in human platelet lysate
}

\author{
Sandra Laner-Plamberger ${ }^{1,2 \dagger}$, Thomas Lener ${ }^{1,2 \dagger}$, Doris Schmid ${ }^{1,2}$, Doris A. Streif ${ }^{1,2}$, Tina Salzer², Michaela Öller ${ }^{1,2}$, \\ Cornelia Hauser-Kronberger ${ }^{3}$, Thorsten Fischer ${ }^{4}$, Volker R. Jacobs ${ }^{4}$, Katharina Schallmoser ${ }^{1,2}$, Mario Gimona ${ }^{1,2}$ \\ and Eva Rohde ${ }^{1,2^{*}}$
}

\begin{abstract}
Background: Pooled human platelet lysate ( $\mathrm{pHPL}$ ) is an efficient alternative to xenogenic supplements for ex vivo expansion of mesenchymal stem cells (MSCs) in clinical studies. Currently, porcine heparin is used in pHPL-supplemented medium to prevent clotting due to plasmatic coagulation factors. We therefore searched for an efficient and reproducible medium preparation method that avoids clot formation while omitting animal-derived heparin.

Methods: We established a protocol to deplete fibrinogen by clotting of pHPL in medium, subsequent mechanical hydrogel disruption and removal of the fibrin pellet. After primary culture, bone-marrow and umbilical cord derived MSCs were tested for surface markers by flow cytometry and for trilineage differentiation capacity. Proliferation and clonogenicity were analyzed for three passages.

Results: The proposed clotting procedure reduced fibrinogen more than 1000-fold, while a volume recovery of $99.5 \%$ was obtained. All MSC types were propagated in standard and fibrinogen-depleted medium. Flow cytometric phenotype profiles and adipogenic, osteogenic and chondrogenic differentiation potential in vitro were independent of MSC-source or medium type. Enhanced proliferation of MSCs was observed in the absence of fibrinogen but presence of heparin compared to standard medium. Interestingly, this proliferative response to heparin was not detected after an initial contact with fibrinogen during the isolation procedure.
\end{abstract}

Conclusions: Here, we present an efficient, reproducible and economical method in compliance to good manufacturing practice for the preparation of MSC media avoiding xenogenic components and suitable for clinical studies.

Keywords: Fibrinogen, Heparin, Mesenchymal stem cells, Pooled human platelet lysate

\section{Background}

The isolation and ex vivo expansion of mesenchymal stem cells (MSCs) is a prerequisite for clinical evaluation regarding their promotion of various therapeutic effects such as tissue regeneration, neuroprotection or immunomodulation $[1,2]$. Cell culture media used for in vitro

\footnotetext{
*Correspondence: e.rohde@salk.at

†Sandra Laner-Plamberger and Thomas Lener contributed equally to this work

${ }^{2}$ Department of Blood Group Serology and Transfusion Medicine, Spinal Cord Injury and Tissue Regeneration Center Salzburg, Paracelsus Medical University, Lindhofstrasse 20-22, 5020 Salzburg, Austria

Full list of author information is available at the end of the article
}

expansion frequently contain fetal bovine serum (FBS) as a source of growth factors. Even though widely applied, major concerns regarding the use of animal serum have emerged: Bovine serum harbors the risk of xenogenic immune reactions. Human MSCs cultured in FBS were shown to generate immune responses in patients receiving MSC-based therapies [3-5]. This might be due to bovine proteins, that were shown to be internalized either directly into the cells [6] or transferred by bovine extracellular vesicles [7]. Furthermore, pathogens such as viruses, mycoplasma and prions can be transmitted by animal sera [8-11]. In addition, bovine sera vary from batch to batch with regard to their effectiveness to 
support cell proliferation [12, 13]. Because of these concerns, the use of FBS in human cell culture applied for stem cell therapies is not recommended to date $[14,15]$. Thus, a growing demand for human alternatives such as pooled human platelet lysate (pHPL) has emerged for clinical trials investigating MSCs [16]. pHPL contains abundant growth factors and cytokines to efficiently boost MSC proliferation in vitro $[17,18]$. Further, pHPL components include plasmatic coagulation factors such as fibrinogen and various platelet-derived factors $[19,20]$. As a result of physiological coagulation, pHPL added to calcium-containing growth media rapidly form viscoelastic fibrin gels. To prevent clot formation in cell culture, heparin, a highly sulfated glycosaminoglycan [21], is commonly added. It has been shown that the concentration and quality of heparin, usually of porcine origin, and the added preservatives therein are critical for cell culture with respect to proliferation, colony forming and ex vivo differentiation capacity of mesenchymal stem cells, but also various other cell types [22-27]. Data further indicate that heparin disrupts the CXCR4/SDF-1 signaling axis and may interfere with migration and homing capacity of BM-derived mononuclear cells [28]. Therefore it has been suggested that heparin should be supplied at lowest possible concentrations in media containing pHPL to prevent gel formation [26]. It may be not only beneficial to avoid heparin, but also to deplete fibrinogen which is a known pro-inflammatory component of pHPL [29]. A recent study demonstrated that fibrinogen increases the adhesion of peripheral blood human natural killer cells, which in turn stimulate human BM-MSC invasion and may not only induce tissue repair but also an exacerbated inflammatory response [29]. Recent data suggest that fibrinogen may negatively affect the immune modulating capacity of MSCs [30]. Considering numerous clinical trials for the treatment of immune disorders, fibrinogen-depletion in PHPL-containing growth media is potentially useful. To date procedures to reduce fibrinogen and to avoid heparin are based on the addition of $\mathrm{CaCl}_{2}$ to undiluted pHPL to antagonize citrate effects and to induce the coagulation cascade, thus producing serum-converted platelet lysate [30,31].

In our study, we searched for alternative methods to deplete fibrinogen in pHPL-supplemented media while preventing the use of any additional reagents such as $\mathrm{CaCl}_{2}$ or porcine heparin. Fibrin polymers rapidly assemble by a modified polycondensation reaction from fibrinogen resulting in three-dimensional networks with large elastic moduli $[32,33]$. Owing to the hydration and the physical properties of viscoelastic fibrin polymer gels, the networks are sensitive to strain and excessive strain burden causes the collapse and aggregation of the fibrin fibers. We have applied a strategy based on this fibrin gel physics to remove fibrinogen from pHPL-based cell culture media without additional requirement for further chemical support. Following our protocol, the resulting complete growth medium remains clear and no further gel or fiber formation is observed. Here we show that heparin free and fibrinogen depleted media are equally efficient to standard pHPL media containing heparin for the cultivation of human MSCs. The here described method is the first to gain entirely humanized cell culture media with high medium recovery rates after induced clotting and low remaining fibrinogen amounts. Thus, it facilitates a standardized and GMP-grade generation of medium for future clinical studies.

\section{Methods}

\section{Medium preparation, fibrinogen depletion and quantification}

Three different media were prepared (medium A, B, C) using alpha-modified Minimum Essential Medium Eagle ( $\alpha$ MEM M4526, Sigma Aldrich, St. Louis, MO, USA) supplemented with $10 \% \mathrm{pHPL}$ and $5.5 \mathrm{mM}$ (N2)L-Alanyl-L-Glutamin (Dipeptiven, Fresenius Kabi, Graz, Austria). pHPL was produced as described with minor modifications [18]. In brief we used outdated buffy-coat derived platelet concentrates (PCs) prepared from regular healthy blood donors at the Department for Transfusion Medicine. For each batch five PCs blood group O were pooled with five PCs blood group A or B. Platelet lysis was induced by several freeze/thaw steps $\left(-30{ }^{\circ} \mathrm{C} / 37^{\circ} \mathrm{C}\right)$ and platelet fragments were depleted by centrifugation $(4000 \times g, 15 \mathrm{~min})$. Suitable aliquots were stored at $-30{ }^{\circ} \mathrm{C}$ until use. Dipeptiven was used due to long-lasting stability in culture medium compared to standard L-Glutamin. Medium A (+fibrinogen/+heparin) was prepared as previously described [18] with $2 \mathrm{IU} / \mathrm{mL}$ of stabilisator-free porcine heparin (Biochrom, Berlin, Germany) to prevent clotting (Fig. 1, white bars) and is referred to as standard medium. Media $B$ and $C$ were produced by intentional hydrogel formation after pHPL supplementation in the absence of heparin. After transferring the supplemented $\alpha M E M$ to $50 \mathrm{~mL}$ conical tubes, hydrogel formation was allowed during an incubation for $4 \mathrm{~h}$ at room temperature (RT) followed by overnight $(\mathrm{o} / \mathrm{n})$ incubation at $4{ }^{\circ} \mathrm{C}$. Finally the coagulated medium was heated to $37^{\circ} \mathrm{C}(1 \mathrm{~h})$ to allow complete fibrin clotting. The collapse after hydrogel formation was induced by vigorous shaking of the conical tubes followed by a centrifugation step $(10 \mathrm{~min}$ at $3000 \times g$, $\mathrm{RT})$. The resulting clear medium supernatant was filtered through a $0.22 \mu \mathrm{m}$ filter (Merck Millipore, Billerica, MA, USA). While medium B (-fibrinogen/-heparin, Fig. 1, grey) remained without further supplement, medium $C$ (-fibrinogen/+heparin, Fig. 1, black) was supplemented with $2 \mathrm{IU} / \mathrm{mL}$ of heparin. After fibrinogen depletion, the 


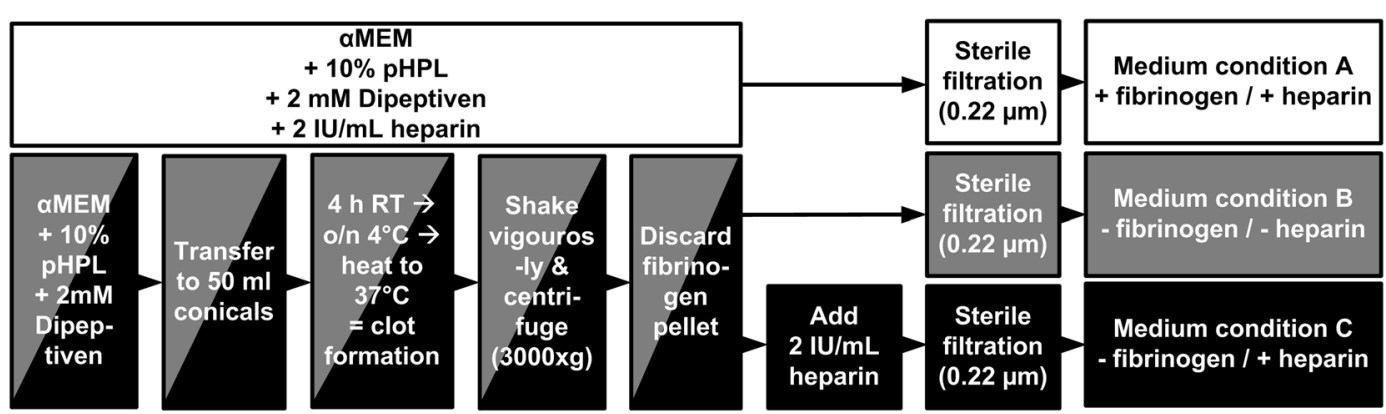

Fig. 1 Production of standard medium (condition A), fibrinogen-depleted medium without (condition B) and with heparin (condition C). To prepare standard medium A (white), aMEM was supplemented with $10 \%$ pooled human platelet lysate (pHPL), $5.5 \mathrm{mM}$ Dipeptiven and $2 \mathrm{IU} / \mathrm{mL}$ heparin. Medium B (grey) and C (black) were prepared by supplementing aMEM with $10 \% \mathrm{pHPL}$ and $5.5 \mathrm{mM}$ Dipeptiven and by allowing clot formation over night $(\mathrm{o} / \mathrm{n})$ followed by mechanical disruption of the fibrin gel by vigorous shaking. After removal of the fibrin pellet medium $\mathrm{B}$ was ready to use, while medium C was additionally supplemented with $2 \mathrm{IU} / \mathrm{mL}$ heparin

volume recovery of media $B$ and $C$ was measured. The concentration of residual fibrinogen was determined in quadruplicate for three independent medium preparations by ELISA (Abcam, Cambridge, UK) according to the manufacturer's instructions.

\section{Experimental setup, isolation and propagation of MSCs}

The study was performed in accordance with the Helsinki Declaration. All donors signed an informed consent concerning the research use of the donated whole blood for pHPL production and of donated umbilical cord (UC) or bone marrow $(\mathrm{BM})$ tissue. UC-MSCs $(\mathrm{n}=10)$ and BM-MSCs $(\mathrm{n}=3)$ were isolated as described previously $[16,34,35]$. Antibiotics $(100 \mathrm{mg} / \mathrm{mL}$ streptomycin and $62.5 \mathrm{mg} / \mathrm{mL}$ penicillin, LifeTechnologies, Carlsbad, CA, USA), were used for initial isolation of UC-MSCs only and removed after the first $48 \mathrm{~h}$. All subsequent culture conditions lacked antibiotics and cells were cultured at $37^{\circ} \mathrm{C}, 5 \% \mathrm{CO}_{2}$ and $95 \%$ humidity.

UC-MSCs of five independent donations (group 1, Fig. 2) were initially isolated using standard medium A only. Another five donations (group 2) were immediately separated into three cord pieces and differentially isolated in either medium condition A, B or C. We further compared the functional response of UC-MSCs to various media types with BM-MSCs (group 3). Because we regularly collected BM-aspirates using heparin, we took BM-MSCs formerly isolated in standard medium A (corresponding to UC-MSCs group1) as controls. After expansion, all MSCs were characterized by flow cytometric analysis and differentiation assays as well as by proliferation and colony forming unit (CFU) assays over three subsequent passages (Fig. 2).

\section{Flow cytometric analysis}

MSCs were incubated in blocking buffer (5\% sheep serum (Eubio, Vienna, Austria) in PBS) in the dark at $4{ }^{\circ} \mathrm{C}$ for $25 \mathrm{~min}$. Cells were stained with fluorescein isothyocyanate (FITC), phycoerythrin (PE) or allophycocyanin (APC)-conjugated mouse anti-human antibodies and corresponding isotype controls (Table 1) in flow cytometry buffer (PBS and $2 \% \mathrm{FBS}$ ) at $4{ }^{\circ} \mathrm{C}$ in the dark for 25 min. Cells were washed, and analyzed using a FC500 flow cytometer (Beckman Coulter, Brea, CA, USA). A minimum of 10,000 nucleated cells was acquired and data were analyzed with FlowJo software (Vers. 7.6; TreeStar Inc., Ashland, OR, USA).

\section{Differentiation assays}

The adipogenic, osteogenic and chondrogenic differentiation potential of group 1 and group 2 UC-MSCs and BM-MSCs (group 3) was tested after expansion in the particular medium type used for the primary isolation (adipogenic differentiation: passage $=2$, osteogenic and chondrogenic differentiation: passage $=3$ ). For osteogenic and adipogenic differentiation, $1000 \mathrm{BM}$ - or $\mathrm{UC}-\mathrm{MSCs} / \mathrm{cm}^{2}$ were seeded. After $24 \mathrm{~h}$, medium was replaced by differentiation medium as described [36]. At day 21, cells were fixed using $4 \%$ paraformaldehyd (PFA, Sigma Aldrich) and stained with either $0.5 \%$ Alizarin Red (Sigma Aldrich) or 1 \% Sudan III (Sigma Aldrich). Chondrogenic differentiation was induced using 250,000 cells per pellet cultivated in hMSC chondrogenic SingleQuots (Lonza, Basel, Switzerland) in the presence of TGF- $\beta 3(20 \mu \mathrm{g} / \mathrm{mL})$ for 21 days. Pellets were harvested by centrifugation $(1500 \times g$ for $5 \mathrm{~min})$, fixed in $4 \%$ PBS buffered formalin and paraffin-embedded. After deparaffination of $2 \mu \mathrm{m}$ sections in graded alcohols, $1 \%$ Alcian Blue staining solution (8GS, GattKoller, Absam, Austria) and Nuclear Fast Red solution (Sigma Aldrich) was applied for 15 min (Multistainer platform, Leica, Wetzlar, Germany). Photographs were taken using a PrimoVert Light microscope equipped 


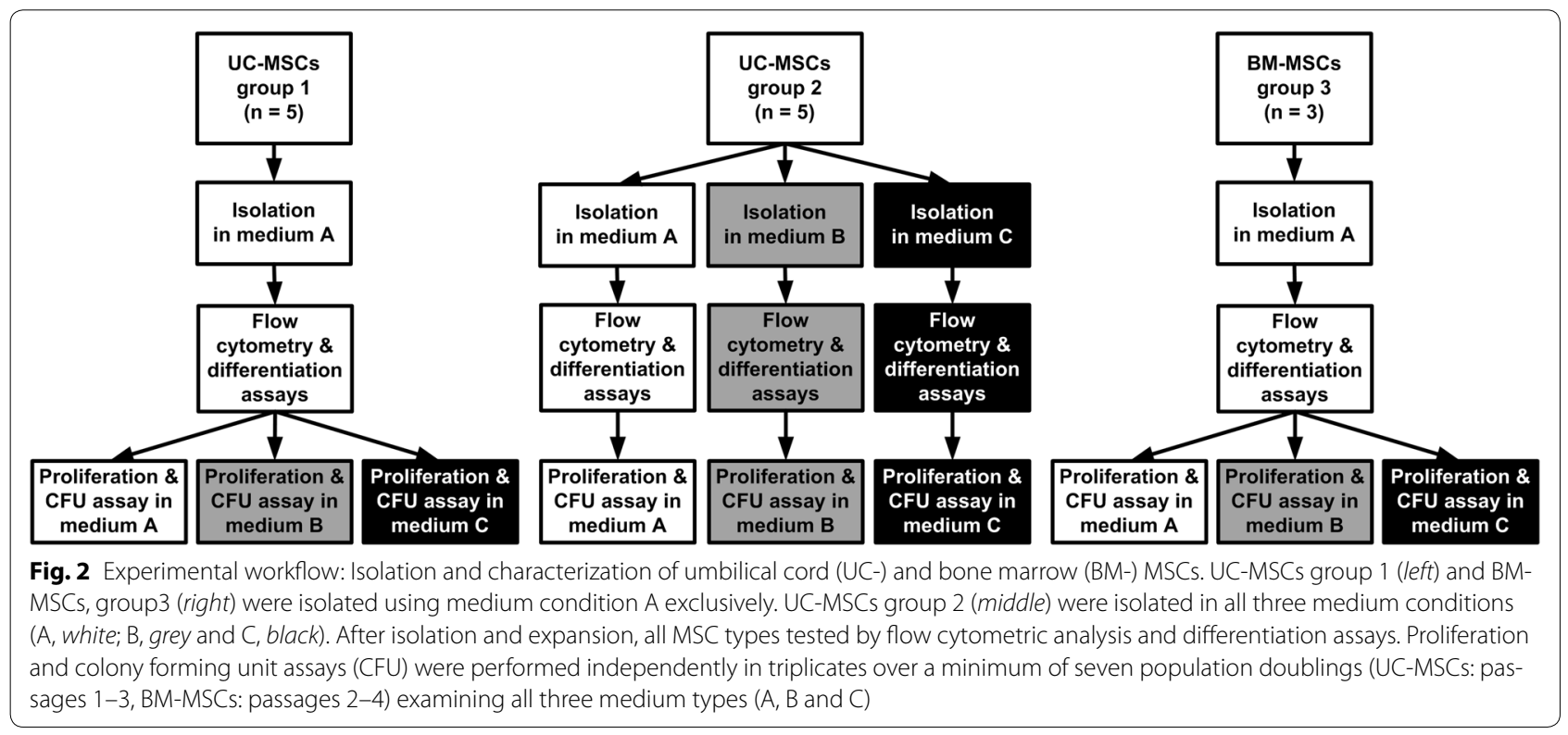

Table 1 Antibodies used for flow cytometric analysis of isolated UC- and BM-MSCs

\begin{tabular}{llll}
\hline Antibody & Conjugate & Company & Clone \\
\hline Anti HLA-DR (MHC II) & FITC & Becton Dickinson, Franklin Lakes, NJ, USA & L243 \\
Anti-h/m/rNG2/MCSP & PE & R\&D Systems, McKinley Place, MN, USA & LHM-2 \\
CD105 & APC & Caltag Laboratories, Carlsbad, CA, USA & MN6 \\
CD14 & PE-Cy7 & Becton Dickinson & 28D4 \\
CD140b & PE & Becton Dickinson & BV9 \\
CD144 (VE-Cadherin) & APC & BioLegend, San Diego, CA, USA & SJ25C1 \\
CD19 & FITC & Becton Dickinson & C40-1457 \\
CD271 & PE & Becton Dickinson & WM59 \\
CD31 & FITC & Becton Dickinson & 8G12 \\
CD34 & PE & Becton Dickinson & HI30 \\
CD45 & BPC & Becton Dickinson & AD2 \\
CD73 & Becton Dickinson & F15-42-1-5 \\
CD90 & PE & Immunotech, Quebec, Canada & X40 \\
Mouse lgG1 & FITC & Becton Dickinson & X40 \\
Mouse lgG1 & FITC & Becton Dickinson & \\
Mouse lgG1 & PE & Becton Dickinson & \\
Mouse lgG2a & APC & Becton Dickinson & \\
\hline
\end{tabular}

with an AxioCam ERc5 s digital camera (both from Zeiss, Oberkochen, Germany).

\section{Proliferation and colony forming unit (CFU) assays}

To test proliferation, $1300 \mathrm{UC}-\mathrm{MSCs} / \mathrm{cm}^{2}$ and 3300 BM-MSCs $/ \mathrm{cm}^{2}$ were seeded in all three medium types. After $96 \mathrm{~h}$, cell number was determined using "Neubauer improved" counting chambers (C-Chip, Biochrom). Cell growth was evaluated by total cell counts and cumulative population doublings (cPD) by means of the formula $\ln (\mathrm{N}) / \ln (2)$, where $\mathrm{N}$ is the cell number of detached cells divided by the number of cells seeded [37]. To investigate colony forming capacity, $3 \mathrm{MSCs} / \mathrm{cm}^{2}$ were seeded in cell culture dishes and cultured for 14 days. Colonies were fixed in $4 \%$ PFA (Sigma Aldrich) and stained with $0.05 \%$ Crystal Violet (Sigma Aldrich). Colonies were counted visually. Each assay was done in triplicate over three subsequent passages (BM-MSCs: passages 2-4, UC-MSCs: passages $1-3)$. 


\section{Statistical analysis}

Data are presented as arithmetic mean \pm standard deviation (SD). Data were compared using 2-way ANOVA and Bonferroni multiple comparison test. Significant results are signed by asterisks $(\mathrm{p}<0.001$ or $\mathrm{p}<0.05)$. Analysis was done with GraphPad Prism 5 (GraphPad Software, La Jolla, CA, USA).

\section{Results}

Mechanical clot depletion results in significant reduction of fibrinogen and high rates of media volume recovery Following hydrogel formation, disruption and centrifugation, the filtration step resulted in a clear and liquid medium without delayed fibrin precipitation over 4 weeks. Measurement of fibrinogen levels in standard (medium A) compared to fibrinogen-depleted media (B and $\mathrm{C}$ ) showed an efficient reduction from $70,935 \mathrm{ng} /$ $\mathrm{mL}$ starting concentration to more than 1000-fold lower fibrinogen levels $(66.2$ and $64.5 \mathrm{ng} / \mathrm{mL}$, respectively, Fig. 3). After clot formation and centrifugation the volume recovery was $99.5 \pm 0.2 \%(n=10)$.

Phenotype and in vitro functionality of human UCand BM-MSCs are independent of fibrinogen and heparin Flow cytometry of UC-MSCs initially isolated in standard medium (group 1), of differentially isolated UC-MSCs (group 2) and of BM-MSCs (group 3) revealed a consistent surface protein expression pattern $\left(\mathrm{CD}^{+} 3^{+} / 90^{+} / 105^{+} /\right.$ NG- $2^{+}$and $\mathrm{CD}^{-} 4^{-} / 19^{-} / 31^{-} / 34^{-} / 45^{-} / 144^{-} / 271^{-} / \mathrm{HLA}-$ $\mathrm{DR}^{-}$) characteristic for MSCs (Fig. $4 \mathrm{a}-\mathrm{c}$ ). Discrete variations in surface marker expression were attributed to donor variability, but were independent of medium conditions during isolation and propagation (Additional file 2: Figure S2). Tri-lineage differentiation potential evaluation showed no major differences between cell and medium types. Following adipogenic induction,

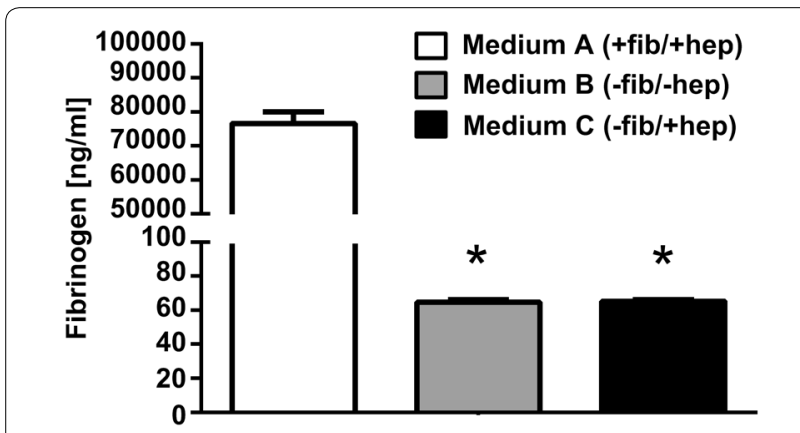

Fig. 3 Quantitative ELISA reveals efficient depletion of fibrinogen. Quantitative ELISA demonstrated that fibrinogen concentration in depleted media B and C is 1000-fold lower compared to standard medium A. Data shown are mean values of three independent medium preparations measured in quadruplicates \pm standard deviation (SD). The significance level is indicated by asterisks ( ${ }^{*} p<0.001$ )
BM-MSCs showed more and larger lipid droplets compared to both UC-MSC groups (Fig. 4d-f, upper panel). All MSCs showed comparable chondrogenic differentiation potential as demonstrated by Alcian Blue staining (Fig. 4d-f, lower panel). No variations in differentiation potential were observed within UC-MSC group 2: independent of medium condition A, B or C UC-MSCs demonstrated comparable tri-lineage differentiation potential (data not shown).

\section{Heparin increases proliferation of UC- MSCs in the absence of fibrinogen}

Proliferation assays performed with the three different medium conditions (A, B or C) revealed no significant differences between the cell counts of UC-MSCs group 1 and BM-MSCs (group 3) that had been exclusively isolated in standard medium A (Fig. 5a and Additional file 1: Figure S1). UC-MSCs (group 2) initially isolated and further propagated either in the presence (medium A) or in the absence of both, fibrinogen and heparin (medium B) showed similar proliferative activity compared to UCMSCs group 1 and BM-MSCs (group 3). After primary isolation of UC-MSCs in medium $\mathrm{C}$, the persistent presence of heparin in fibrinogen-depleted medium resulted in a sustained and pronounced proliferative response (Fig. 5a and Additional file 1: Figure S1). These results were partially mirrored in CFU assays (Fig. 5b, c). While the total cell counts reached similar levels as compared to UC-MSCs group 1 the corresponding CFU numbers were reduced in UC-MSC group 2 propagated in standard medium A (fib $+/$ hep + ). A similar, but more pronounced effect was observed for BM-MSCs: While colony number was reduced, the total cell count was comparable with UC-MSC group 1. This could be attributed to the high proliferative activity of single clones resulting in larger colony sizes (and higher cell numbers per colony). One obvious difference was the consistently weak Crystal Violet staining that was found with BM-MSCs as compared to UC-MSCs. However cell density per colony was high, thus MSC counts reached comparable levels to UC-MSC group 1 (Fig. 5c).

These data point to a significant mitogenic effect of heparin on primary UC-MSCs, when isolated and permanently cultured in the absence of fibrinogen. However, if MSCs had former contact with fibrinogen (UC-MSC group 1 and BM-MSC, group 3) this response to heparin was not observed, suggesting that the mitogenic effect of heparin might be masked by fibrinogen.

\section{Discussion}

Human MSCs are key candidates for cell therapy with the intention to treat immune disorders like graft-versus-host disease, or multiple sclerosis, inherited tissue 


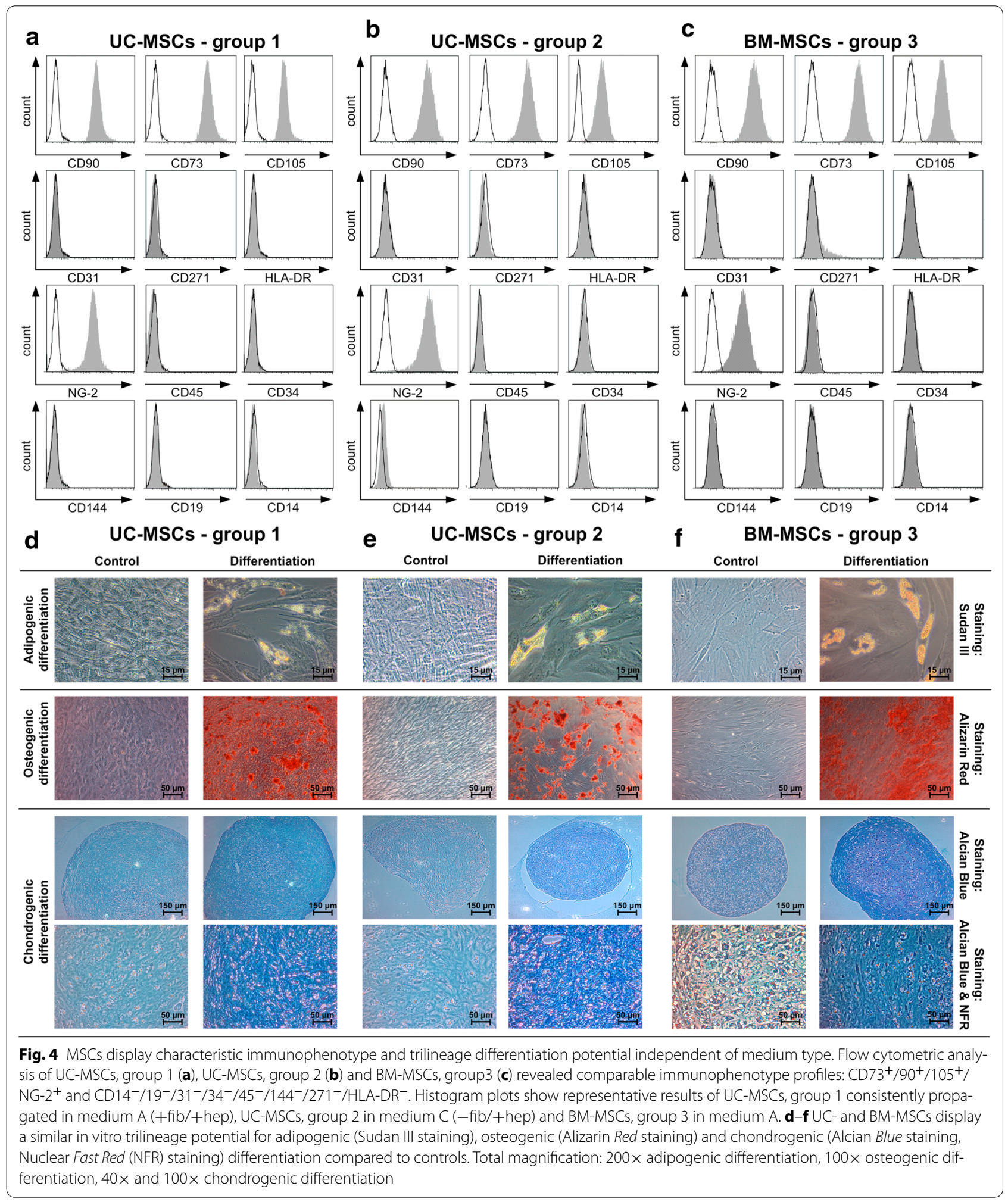

defects such as osteogenesis imperfecta and traumatic, toxic or ischemic organ damage [38-41]. Animal components are not recommended to be used in clinical trials and can be replaced by pHPL derived from human platelet rich plasma (PRP) [17, 18]. To avoid unintended clot formation of plasmatic coagulation factors 


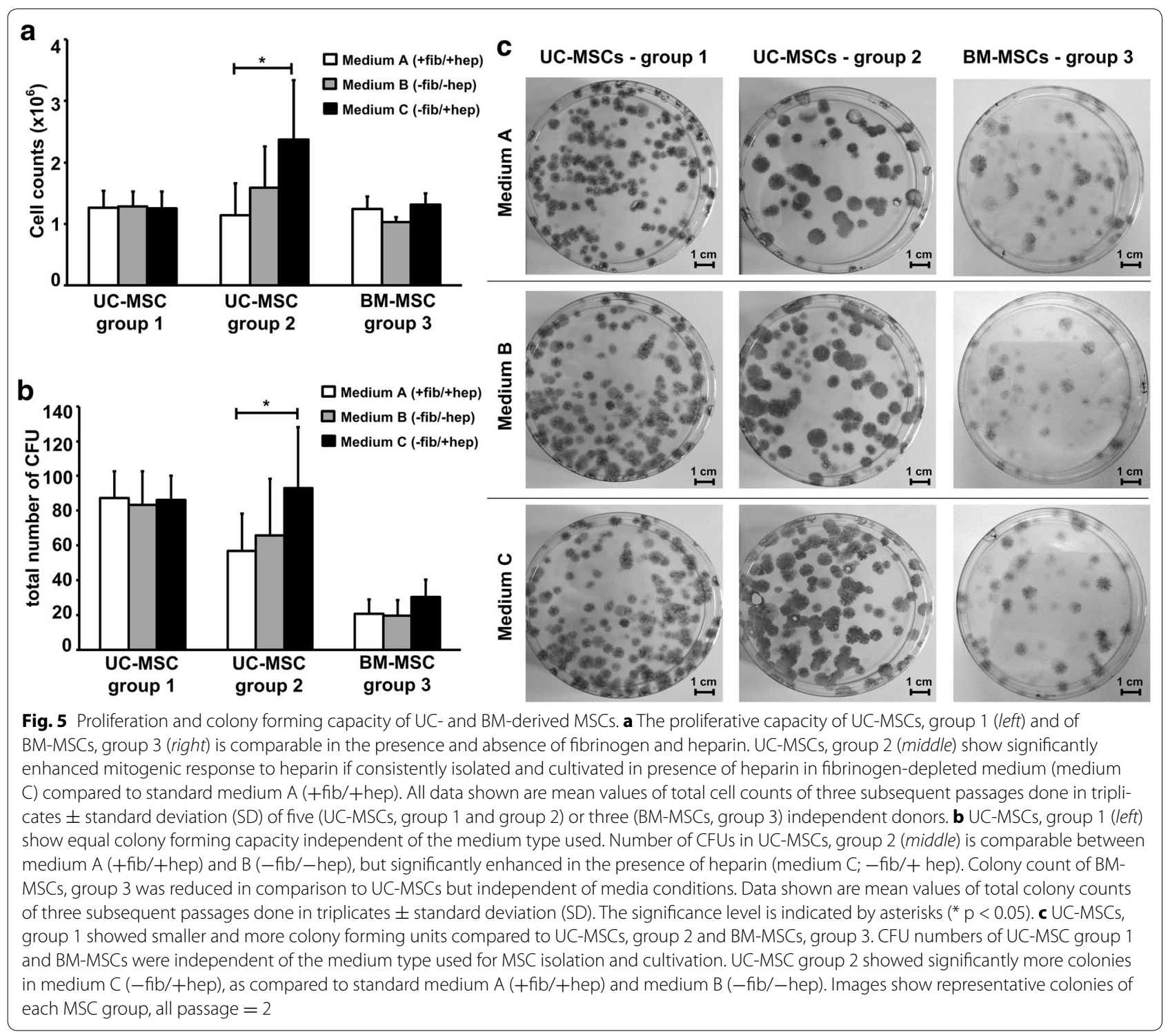

in pHPL-supplemented medium, the addition of porcine-derived heparin is commonly used. Although porcine heparin has been clinically applied for decades, some reports have pointed out severe side effects such as thrombocytopenia, hyperkalemia and hypersensitivity in up to $5 \%$ of the patients, which is ascribed either to the product itself, the impurities contained within the product or the type of preparation [42-48]. Attempts to produce heparin by chemoenzymatic synthesis have been reported and may help to avoid the porcine product in human cell culture [49]. However, even if synthesized successfully it would be necessary to demonstrate similar in vivo and in vitro effects of synthetic heparin on MSC functionality, such as immunomodulatory potential, stemness and proliferative, migratory as well as homing capacity $[28,30,50,51]$.
In order to avoid the addition of heparin, work from Copland et al. and Mojica-Henshaw et al. suggested the reduction of heparin and also of fibrinogen from complete cell culture growth media formulations [30, 31]. Their proposed strategy used forced gelation of platelet lysate by addition of $\mathrm{CaCl}_{2}$ prior to cell culture medium supplementation. This method may be limited by (1) nonphysiologic calcium levels, that in turn may negatively influence the osteogenic properties of MSCs [52], and (2) by poor volume recovery of fibrinogen-depleted platelet lysate. Only $60 \%$ of the HPL starting volume was recovered after the clotting procedure by Copland et al. [30]. The method presented in this study allows clot formation of pHPL supplemented medium and achieves a $99.5 \%$ medium recovery rate, while achieving a more than $1000-$ fold fibrinogen reduction. In contrast to protocols that 
suggest a minimum concentration of heparin (0.6 IU/ $\mathrm{mL}$ ) [26], our heparin-free method represents a robust procedure resulting in a clear cell culture medium without delayed further clotting or fibrin precipitation. The method can be performed in a highly standardized manner, omits the addition of nonessential reagents, facilitates an economic use of pHPL as human alternative to FBS and thus provides an efficient and reproducible preparation of complete cell culture growth medium.

pHPL as an alternative to FBS is increasingly used in the scientific community and is under investigation as an important supplement in fully humanized culture systems for the generation of cellular therapeutics. It is regarded as rather safe concerning infectious diseases due to the routinely tested infectious parameters (for most European blood centers: HIV1/2, HAV, HBV, HCV, TPHA and Parvo B19). However, there is a certain risk of transmitting viruses that are not routinely tested in blood donors. Therefore, in 2014 Castiglia et al. suggested to subject pHPL to pathogen inactivation (iHPL) by psoralen and showed that there were no significant differences between pHPL and iHPL concerning colony-forming unit number, immunophenotype or multipotent capacity of BM-MSCs [53]. However, the effect of psoralen on MSCs expanded in iHPL has not yet been investigated in more detail. Further studies are required to demonstrate the role of psoralen in cell culture of MSCs for clinical applications. In addition, there are reports about patients with allergic reactions to psoralen [54]. In order to circumvent psoralen, pathogen inactivation can also be done with riboflavin in combination with UVA or using UVC only. However, several studies show that all of the pathogen inactivating treatments influence the proteome and activation states of platelets $[55,56]$. This might impact on functionality of cell therapy products generated in iHPL. As an alternative, quality tests for a broad range of contaminating viruses, which are performed on a non-routine basis, are discussed to ensure safety and purity of cell therapy products [57]. However, results of a risk-based analysis performed in our cell production facility, an academic GMP-laboratory, did not argue for extended virus testing.

Analysis of the different media formulations for proliferative and functional support of UC-MSCs and BM-MSCs revealed no significant differences in proliferation, colony forming capacity, phenotype and in vitro tri-lineage differentiation, if MSCs were isolated in the presence or absence of both, fibrinogen and heparin. In CFU assays we found higher colony numbers in UC-MSCs group 1 as compared to group 2 in standard medium, a difference which was not reflected in absolute cell counts. This effect can be assigned to donor variability with large size and enhanced cell density per colony compensating the observed difference in CFU numbers. Our findings support the assumption that fibrinogen-depletion does not result in a significant loss of various pHPL-supplied growth factors or components critical for adipo-, osteo- and chondrogenic induction. Therefore fibrinogen-depleted and heparin-free medium is suitable for the successful in vitro propagation of functional MSCs, suggesting that both substances are dispensable for the effective expansion of UC- and BM-MSCs.

However, we provide evidence that during isolation and further expansion of UC-MSCs, fibrinogen and heparin do critically influence at least proliferative capacity. UC-MSCs of the same donor primarily isolated and maintained in the absence of fibrinogen, but in the presence of heparin, showed an enhanced proliferation compared to UC-MSCs initially isolated in standard medium. This proliferative response to heparin was never found after an initial contact to fibrinogen. The enhanced proliferative effect of heparin on UC-MSCs isolated and cultivated in fibrinogen-depleted medium indicates a strong mitogenic stimulus induced by heparin, which may be masked by fibrinogen. These results are in line with data suggesting that heparin may interfere with proliferation and functional capacity of MSCs [23, 26, 28, 50]. Further studies are needed to investigate the molecular mechanism and a so far unknown interplay between fibrinogen, heparin and MSCs in vitro as well as putative effects on MSC functionality in vivo.

\section{Conclusion}

In conclusion, we have shown that porcine heparin is dispensable for the propagation of MSCs from umbilical cord and bone marrow. We have developed a convenient, reproducible and GMP-compliant procedure to deplete pHPL-derived fibrinogen in MSC culture medium that can be efficiently used for MSC propagation in clinical trials.

\section{Additional files}

\footnotetext{
Additional file 1: Figure S1. MSC proliferation shown as cumulative population doublings (CPD). CPD have been calculated. The proliferative capacity of UC-MSCs, group 1 and of BM-MSCs, group 3 is comparable in the presence and absence of fibrinogen and heparin. In contrast, CPD of group 2 UC-MSCs show a significantly enhanced proliferation in response to heparin (group 2_C) compared to medium A and medium B. Data shown are mean values of CPD of three passages done in triplicates \pm standard deviation (SD) of five (UC-MSCs) or three (BM-MSCs) independent donors.

Additional file 2: Figure S2. Flow cytometric analysis of individual MSC donations. Flow cytometric analysis of all individual donations of UC-MSCs, group 1 (A), UC-MSCs, group 2 (B) and BM-MSCs, group 3 (C). All MSCs isolated show a characteristic immunophenotype: $\mathrm{CD}^{+} 3^{+} / 90^{+} / 105^{+} / \mathrm{NG}^{-} 2^{+}$and $\mathrm{CD} 14^{-} / 19^{-} / 31^{-} / 34^{-} / 45^{-} / 144^{-} / 271^{-} /$ HLA-DR ${ }^{-}$. No significant differences in surface marker expression could be observed if MSCs of the same donor were cultivated in different medium conditions ( $A, B$ or $C$ ); Minor variations in expression profiles were ascribed to donor variability.
} 


\section{Abbreviations}

BM: bone marrow; BM-MSCs: bone marrow-derived mesenchymal stem cells: Fib: fibrinogen; GMP: good manufacturing practice; Hep: heparin; MSCs: Mesenchymal stem cells; PC: platelet concentrate; pHPL: pooled human platelet Iysate; UC: umbilical cord; UC-MSCs: umbilical cord-derived mesenchymal stem cells.

\section{Authors' contributions}

SLP participated in the design of the study, carried out the isolation of UCMSCs, performed the corresponding proliferation and CFU assays and all differentiation assays (UC-MSCs and BM-MSCs), did statistical analysis and drafted the manuscript. TL participated in the design of the study, carried out proliferation and CFU assays regarding BM-MSCs and drafted the manuscript. SLP and TL did the analysis and interpretation of the data. DS performed flow cytometry and participated in analysis and interpretation of the data. DAS participated in the isolation of UC-MSCs, performed proliferation and CFU assays regarding BM-MSCs and did the statistical analysis. TS participated in MSC isolation and flow cytometry. MÖ did flow cytometry, provided pHPL and participated in data analysis. CHK performed histochemical staining and analysis of chondrogenic differentiation assays. TF and VRJ participated in study design, coordination of tissue donations and critically revised the manuscript. KS provided pHPL, participated in data interpretation and critically revised the manuscript. MG participated in study design, performed the isolation of BM-MSCs, did analysis and interpretation of the data, drafted and critically revised the manuscript. ER participated in the study design and interpretation of the data, drafted the manuscript and gave final approval of the version to be published. All authors read and approved the final manuscript.

\section{Author details}

${ }^{1}$ Spinal Cord Injury and Tissue Regeneration Center Salzburg, Paracelsus Medical University, Salzburg, Austria. ${ }^{2}$ Department of Blood Group Serology and Transfusion Medicine, Spinal Cord Injury and Tissue Regeneration Center Salzburg, Paracelsus Medical University, Lindhofstrasse 20-22, 5020 Salzburg, Austria. ${ }^{3}$ Department of Pathology, Paracelsus Medical University, Salzburg, Austria. ${ }^{4}$ Department of Gynecology and Obstetrics, Paracelsus Medical University, Salzburg, Austria.

\section{Acknowledgements}

We thank Prof. Dr. Dirk Strunk (Institute of Experimental und Clinical Cell Therapy, Spinal Cord Injury and Tissue Regeneration Center Salzburg (SCI-TReCS), Paracelsus Medical University, Salzburg) and Dr. Andreas Reinisch (Institute for Stem Cell Biology and Regenerative Medicine (ISCBRM), Stanford University, CA) for fruitful discussion, the midwife team of the Department of Gynecology and Obstetrics, Paracelsus Medical University, Salzburg, for their support and Nina Held, Nicole Kerschbaumer and Brian van Merkestijen for excellent technical assistance. This work was supported by the Spinal Cord Injury and Tissue Regeneration Center Salzburg (SCI-TReCs).

\section{Competing interests}

The authors declare that they have no competing interests.

Received: 27 August 2015 Accepted: 29 October 2015

Published online: 10 November 2015

\section{References}

1. Wang S, Qu X, Zhao RC (2012) Clinical applications of mesenchymal stem cells. J Hematol Oncol 5:19. doi: 10.1186/1756-8722-5-19 (1756-8722-5-19 [pii]).

2. Glenn JD, Whartenby KA. Mesenchymal stem cells: emerging mechanisms of immunomodulation and therapy. World J Stem Cells. 2014;6(5):526-39. doi:10.4252/wjsc.v6.i5.526.

3. Selvaggi TA, Walker RE, Fleisher TA. Development of antibodies to fetal calf serum with arthus-like reactions in human immunodeficiency virus-infected patients given syngeneic lymphocyte infusions. Blood. 1997:89(3):776-9.

4. Mackensen A, Drager R, Schlesier M, Mertelsmann R, Lindemann A. Presence of IgE antibodies to bovine serum albumin in a patient developing anaphylaxis after vaccination with human peptide-pulsed dendritic cells. Cancer Immunol Immunother. 2000;49(3):152-6.
5. Tuschong L, Soenen SL, Blaese RM, Candotti F, Muul LM. Immune response to fetal calf serum by two adenosine deaminase-deficient patients after T cell gene therapy. Hum Gene Ther. 2002;13(13):1605-10. doi:10.1089/10430340260201699.

6. Spees JL, Gregory CA, Singh H, Tucker HA, Peister A, Lynch PJ, et al. Internalized antigens must be removed to prepare hypoimmunogenic mesenchymal stem cells for cell and gene therapy. Mol Ther. 2004;9(5):747-56. doi:10.1016/j.ymthe.2004.02.012.

7. Shelke GV, Lasser C, Gho YS, Lotvall J. Importance of exosome depletion protocols to eliminate functional and RNA-containing extracellular vesicles from fetal bovine serum. J Extracell Vesicles. 2014;3. doi:10.3402/ jev.v3.24783 24783 [pii].

8. Nuttall PA, Luther PD, Stott EJ. Viral contamination of bovine foetal serum and cell cultures. Nature. 1977;266(5605):835-7.

9. Tonti GA, Mannello F. From bone marrow to therapeutic applications: different behaviour and genetic/epigenetic stability during mesenchymal stem cell expansion in autologous and foetal bovine sera? Int J Dev Biol. 2008;52(8):1023-32. doi:10.1387/ijdb.082725gt.

10. Kozasa T, Aoki H, Nakajima N, Fukusho A, Ishimaru M, Nakamura S. Methods to select suitable fetal bovine serum for use in quality control assays for the detection of adventitious viruses from biological products. Biologicals. 2011;39(4):242-8. doi:10.1016/j.biologicals.2011.06.001.

11. Nikfarjam L, Farzaneh P. Prevention and detection of Mycoplasma contamination in cell culture. Cell J. 2012;13(4):203-12.

12. Brunner D, Frank J, Appl H, Schoffl H, Pfaller W, Gstraunthaler G. Serumfree cell culture: the serum-free media interactive online database. Altex. 2010;27(1):53-62.

13. Gstraunthaler G, Lindl T, van der Valk J. A plea to reduce or replace fetal bovine serum in cell culture media. Cytotechnology. 2013;65(5):791-3. doi:10.1007/s10616-013-9633-8.

14. Guideline on the use of bovine serum in the manufacture of human biological medicinal products. EMA/CHMP/BWP/457920/2012rev1 Committee for Medicinal Products for Human Use (CHMP); 2013.

15. Note for guidance on minimising the risk of transmitting animal spongiform encephalopathy agents via human and veterinary medicinal products. EMA/410/01rev3, 2011/C 73; 2011.

16. Schallmoser K, Rohde E, Reinisch A, Bartmann C, Thaler D, Drexler C, et al. Rapid large-scale expansion of functional mesenchymal stem cells from unmanipulated bone marrow without animal serum. Tissue Eng Part C Method. 2008;14(3):185-96. doi:10.1089/ten.tec.2008.0060.

17. Doucet C, Ernou I, Zhang Y, Llense JR, Begot L, Holy X, et al. Platelet lysates promote mesenchymal stem cell expansion: a safety substitute for animal serum in cell-based therapy applications. J Cell Physiol. 2005;205(2):22836. doi:10.1002/jcp.20391.

18. Schallmoser K, Bartmann C, Rohde E, Reinisch A, Kashofer K, Stadelmeyer $E$, et al. Human platelet lysate can replace fetal bovine serum for clinicalscale expansion of functional mesenchymal stromal cells. Transfusion. 2007;47(8):1436-46. doi:10.1111/j.1537-2995.2007.01220.x (TRF01220 [pii]).

19. Bieback K, Hecker A, Kocaomer A, Lannert H, Schallmoser K, Strunk D, et al. Human alternatives to fetal bovine serum for the expansion of mesenchymal stromal cells from bone marrow. Stem Cell. 2009;27(9):233141. doi:10.1002/stem.139.

20. Kinzebach S, Dietz L, Kluter H, Thierse HJ, Bieback K. Functional and differential proteomic analyses to identify platelet derived factors affecting ex vivo expansion of mesenchymal stromal cells. BMC Cell Biol. 2013;14:48. doi:10.1186/1471-2121-14-48 1471-2121-14-48 [pii].

21. Casu B, Lindahl U. Structure and biological interactions of heparin and heparan sulfate. Adv Carbohydr Chem Biochem. 2001;57:159-206.

22. Spivak-Kroizman T, Lemmon MA, Dikic I, Ladbury JE, Pinchasi D, Huang J, et al. Heparin-induced oligomerization of FGF molecules is responsible for FGF receptor dimerization, activation, and cell proliferation. Cell. 1994;79(6):1015-24. doi:10.1016/0092-8674(94)90032-9.

23. Flint N, Cove FL, Evans GS. Heparin stimulates the proliferation of intestinal epithelial cells in primary culture. J Cell Sci. 1994;107(Pt 2):401-11.

24. Wu WK, Shin VY, Ye YN, Wong HP, Huang FY, Hui MK, et al. Heparin increases human gastric carcinoma cell growth. Anticancer Res. 2006;26(1A):439-43.

25. Thornton SC, Mueller SN, Levine EM. Human endothelial cells: use of heparin in cloning and long-term serial cultivation. Science. 1983;222(4624):623-5. 
26. Hemeda H, Kalz J, Walenda G, Lohmann M, Wagner W. Heparin concentration is critical for cell culture with human platelet lysate. Cytotherapy. 2013;15(9):1174-81. doi:10.1016/j.jcyt.2013.05.006 (S14653249(13)00516-1 [pii]).

27. Hemeda H, Giebel B, Wagner W. Evaluation of human platelet lysate versus fetal bovine serum for culture of mesenchymal stromal cells. Cytotherapy. 2014;16(2):170-80. doi:10.1016/j.jcyt.2013.11.004 (S14653249(13)00777-9 [pii]).

28. Seeger FH, Rasper T, Fischer A, Muhly-Reinholz M, Hergenreider E, Leistner DM, et al. Heparin disrupts the CXCR4/SDF-1 axis and impairs the functional capacity of bone marrow-derived mononuclear cells used for cardiovascular repair. Circ Res. 2012;111(7):854-62. doi:10.1161/ CIRCRESAHA.112.265678.

29. Almeida CR, Vasconcelos DP, Goncalves RM, Barbosa MA. Enhanced mesenchymal stromal cell recruitment via natural killer cells by incorporation of inflammatory signals in biomaterials. J R Soc Interface. 2012;9(67):26171. doi:10.1098/rsif.2011.0357

30. Copland IB, Garcia MA, Waller EK, Roback JD, Galipeau J. The effect of platelet lysate fibrinogen on the functionality of MSCs in immunotherapy. Biomaterials. 2013;34(32):7840-50. doi:10.1016/j.biomaterials.2013.06.050 (S0142-9612(13)00765-5 [pii]).

31. Mojica-Henshaw MP, Jacobson P, Morris J, Kelley L, Pierce J, Boyer $M$, et al. Serum-converted platelet lysate can substitute for fetal bovine serum in human mesenchymal stromal cell cultures. Cytotherapy. 2013;15(12):1458-68. doi:10.1016/j.jcyt.2013.06.014 (S14653249(13)00598-7 [pii]).

32. Janmey PA. Kinetics of formation of fibrin oligomers. I Theor Biopolym 1982;21(11):2253-64. doi:10.1002/bip.360211112.

33. Janmey PA, Schliwa M. Rheology. Curr Biol. 2008;18(15):R639-41. doi:10.1016/j.cub.2008.05.001.

34. Reinisch A, Strunk D. Isolation and animal serum free expansion of human umbilical cord derived mesenchymal stromal cells (MSCs) and endothelial colony forming progenitor cells (ECFCs). J Vis Exp. 2009;32:1525. doi:10.3791/1525.

35. Rohban R, Reinisch A, Etchart N, Schallmoser K, Hofmann NA, Szoke $K$, et al. Identification of an effective early signaling signature during neo-vasculogenesis in vivo by ex vivo proteomic profiling. PLoS One. 2013;8(6):e66909. doi:10.1371/journal.pone.0066909.

36. Reinisch A, Etchart N, Thomas D, Hofmann NA, Fruehwirth M, Sinha S, et al. Epigenetic and in vivo comparison of diverse MSC sources reveals an endochondral signature for human hematopoietic niche formation. Blood. 2015;125(2):249-60. doi:10.1182/blood-2014-04-572255 (blood2014-04-572255 [pii])

37. Dell'Orco RT, Mertens JG, Kruse PF Jr. Doubling potential, calendar time, and senescence of human diploid cells in culture. Exp Cell Res. 1973;77(1):356-60.

38. Connick P, Kolappan M, Crawley C, Webber DJ, Patani R, Michell AW, et al. Autologous mesenchymal stem cells for the treatment of secondary progressive multiple sclerosis: an open-label phase $2 \mathrm{a}$ proof-of-concept study. Lancet Neurol. 2012;11(2):150-6. doi:10.1016/ S1474-4422(11)70305-2

39. Hare JM, Traverse JH, Henry TD, Dib N, Strumpf RK, Schulman SP, et al. A randomized, double-blind, placebo-controlled, dose-escalation study of intravenous adult human mesenchymal stem cells (prochymal) after acute myocardial infarction. J Am Coll Cardiol. 2009;54(24):2277-86. doi:10.1016/j.jacc.2009.06.055.

40. Le Blanc K, Frassoni F, Ball L, Locatelli F, Roelofs H, Lewis I, et al. Mesenchymal stem cells for treatment of steroid-resistant, severe, acute graftversus-host disease: a phase II study. Lancet. 2008;371(9624):1579-86. doi:10.1016/S0140-6736(08)60690-X

41. Otsuru S, Gordon PL, Shimono K, Jethva R, Marino R, Phillips CL, et al. Transplanted bone marrow mononuclear cells and MSCs impart clinical benefit to children with osteogenesis imperfecta through different mechanisms. Blood. 2012;120(9):1933-41. doi:10.1182/ blood-2011-12-400085.

42. Bottio T, Pittarello G, Bonato R, Fagiolo U, Gerosa G. Life-threatening anaphylactic shock caused by porcine heparin intravenous infusion during mitral valve repair. J thorac cardiovasc surg. 2003;126(4):1194-5. doi:10.1016/S0022.
43. Harada A, Tatsuno K, Kikuchi T, Takahashi Y, Sai S, Murakami Y, et al. Use of bovine lung heparin to obviate anaphylactic shock caused by porcine gut heparin. Ann thorac surg. 1990;49(5):826-7.

44. Huang Q, Xu T, Wang GY, Huang JF, Xia H, Yin R, et al. Species-specific identification of ruminant components contaminating industrial crude porcine heparin using real-time fluorescent qualitative and quantitative PCR. Anal Bioanal Chem. 2012;402(4):1625-34. doi:10.1007/ s00216-011-5590-2.

45. Liu H, Zhang Z, Linhardt RJ. Lessons learned from the contamination of heparin. Nat Prod Rep. 2009;26(3):313-21. doi:10.1039/b819896a.

46. Gheno G, Savarino C, Vellar S, Cinetto L. Heparin-induced life-threatening hyperkalemia. Ann Ital Med Int. 2002;17(1):51-3.

47. Linkins LA. Heparin induced thrombocytopenia. Bmj. 2015;350:g7566. doi:10.1136/bmj.g7566.

48. Blossom DB, Kallen AJ, Patel PR, Elward A, Robinson L, Gao G, et al. Outbreak of adverse reactions associated with contaminated heparin. $\mathrm{N}$ Engl J Med. 2008;359(25):2674-84. doi:10.1056/NEJMoa0806450.

49. Farrugia BL, Lord MS, Melrose J, Whitelock JM. Can We Produce Heparin/ Heparan Sulfate Biomimetics Using "Mother-Nature" as the Gold Standard? Molecules. 2015;20(3):4254-76. doi:10.3390/molecules20034254.

50. Barak H, Huh SH, Chen S, Jeanpierre C, Martinovic J, Parisot M, et al. FGF9 and FGF20 maintain the stemness of nephron progenitors in mice and man. Dev Cell. 2012;22(6):1191-207. doi:10.1016/j.devcel.2012.04.018 (S1534-5807(12)00195-5 [pii]).

51. Ho W, Tawil B, Dunn JC, Wu BM. The behavior of human mesenchymal stem cells in 3D fibrin clots: dependence on fibrinogen concentration and clot structure. Tissue Eng. 2006;12(6):1587-95. doi:10.1089/ ten.2006.12.1587.

52. Kanatani M, Sugimoto T, Kanzawa M, Yano S, Chihara K. High extracellular calcium inhibits osteoclast-like cell formation by directly acting on the calcium-sensing receptor existing in osteoclast precursor cells. Biochem Biophys Res Commun. 1999;261(1):144-8. doi:10.1006/bbrc.1999.0932 (S0006-291X(99)90932-4 [pii])

53. Castiglia S, Mareschi K, Labanca L, Lucania G, Leone M, Sanavio F, et al. Inactivated human platelet lysate with psoralen: a new perspective for mesenchymal stromal cell production in good manufacturing practice conditions. Cytotherapy. 2014;16(6):750-63. doi:10.1016/j. jcyt.2013.12.008.

54. Walker D, Jacobe H. Phototherapy in the age of biologics. Semin Cutan Med Surg. 2011;30(4):190-8. doi:10.1016/j.sder.2011.08.004.

55. Kaiser-Guignard J, Canellini G, Lion N, Abonnenc M, Osselaer JC, Tissot JD. The clinical and biological impact of new pathogen inactivation technologies on platelet concentrates. Blood Rev. 2014;28(6):235-41. doi:10.1016/j.blre.2014.07.005.

56. Prudent M, D'Alessandro A, Cazenave JP, Devine DV, Gachet C, Greinacher $A$, et al. Proteome changes in platelets after pathogen inactivationan interlaboratory consensus. Transfus Med Rev. 2014;28(2):72-83. doi:10.1016/j.tmrv.2014.02.002.

57. Gombold J, Karakasidis S, Niksa P, Podczasy J, Neumann K, Richardson J, et al. Systematic evaluation of in vitro and in vivo adventitious virus assays for the detection of viral contamination of cell banks and biological products. Vaccine. 2014;32(24):2916-26. doi:10.1016/j.vaccine.2014.02.021.

\section{Submit your next manuscript to BioMed Central and take full advantage of:}

- Convenient online submission

- Thorough peer review

- No space constraints or color figure charges

- Immediate publication on acceptance

- Inclusion in PubMed, CAS, Scopus and Google Scholar

- Research which is freely available for redistribution

Submit your manuscript at www.biomedcentral.com/submit
( Biomed Central 